Gimol Benzaquen Perosa ${ }^{1}$

Isabela Cristina Canavez ${ }^{2}$

Flávia Cristina Pereira Silveira ${ }^{3}$

Flávia Helena Pereira Padoviani ${ }^{4}$

josé Carlos Peraçol ${ }^{5}$

Artigo original

Palavras chave

Depressão

Ansiedade materna

Malformações

Saúde mental

Keywords

Depression

Maternal anxiety

Congenital abnormalities

Mental health

\section{Sintomas depressivos e ansiosos em mães de recém-nascidos com e sem malformações}

\author{
Depressive and anxious symptoms in mothers of newborns with and \\ without malformations
}

\section{Resumo}

OBJETIVO: comparar as condições emocionais de mães cujos filhos nascem com malformações visíveis (Grupo M) com as das mães de crianças eutróficas (Grupo E) logo após o nascimento. MÉTODO: foram avaliados os sintomas de ansiedade e depressão de 22 mães de cada grupo por meio do Inventário de Depressão de Beck (BDI) e do Inventário de Ansiedade Traço-Estado (IDATE). Foram excluídas as mães portadoras de deficiência sensorial incapacitante, HIV, distúrbios psiquiátricos e síndromes genéticas. Os dados foram complementados com consultas a prontuários médicos da criança e da mãe. Para análise comparativa entre as medianas dos grupos foi utilizado o teste não-paramétrico $U$ de Mann-Whitney; para amostras independentes e para os escores indicativos de sintomas clínicos, o teste exato de Fisher e o teste do $\chi^{2}$. RESULTADOS: houve diferenças significativas nas medianas dos escores das três subescalas (ansiedade-traço, ansiedade-estado e disforia/depressão) entre os dois grupos de mães. Houve uma porcentagem significativamente maior de mães do Grupo M com escores indicativos de sinais clínicos para depressão ou ansiedade no pós-parto imediato e, para ambos os quadros, quando comparadas com mães do Grupo E. Os resultados podem ser decorrentes de traços de personalidade materna, visto que os índices de ansiedade-traço eram significativamente maiores nas mães de crianças malformadas, mas especialmente pelo estado da criança, seu encaminhamento para a UTI e sua condição de vida futura. CONCLUSÕES: a porcentagem de mães de recém-nascidos com malformações visíveis que apresentou escores indicativos de sinais clínicos para ansiedade, depressão e ambos sugerem a necessidade de suporte, individual ou em grupo.

\section{Abstract}

PURPOSE: the main goal of this study is to evaluate the emotional conditions among mothers of newborns with visible malformation (Group M) and mothers of eutrophic newborns (Group E) soon after birth. METHODS: twenty-two mothers from the Group M were matched by age and number of children to 22 mothers of the Group E. They were assessed through the Beck Depression Inventory (BDI) and the State-Trait Anxiety Inventory (STAII. The data were complemented by interviews and analyses of the medical files of both mother and child. RESULTS: the results have shown significant differences in the mean scores of the three subscales (trait anxiety, state anxiety, dysphoria/depression) between the two groups of mothers. In terms of clinical signs, there were a significantly larger percentage of mothers from Group M identified with depression and anxiety after the child's birth, and for both conditions when compared with mothers from Group E. Although the results may reflect characteristics of the maternal personality, the trait anxiety was significantly more evident in mothers of malformed children, especially due to the child's health condition, its referral to the ICU and his/her condition regarding their future life. CONCLUSION: the percentage of mothers with clinical depression and anxiety suggests the need for either individual or group support to attend the needs of the mothers and mitigate the adverse effects of stressors throughout the child's development. Support should also be provided during pregnancy, when the mothers currently receive the news about the malformation.
Faculdade de Medicina de Botucatu da Universidade Estadual "Júlio de Mesquita Filho" - UNESP - Botucatu (SP), Brasil.

Professora-assistente do Departamento de Neurologia, Psicologia e Psiquiatria da Faculdade de Medicina de Botucatu da Universidade Estadual Paulista "Júlio de Mesquita Filho" - UNESP - Botucatu (SP), Brasil.

2 Residente do Programa de Residência Médica da Faculdade de Medicina de Ribeirão Preto da Universidade de São Paulo - USP Ribeirão Preto (SP), Brasil.

${ }^{3}$ Mestre em Saúde Coletiva pela Faculdade de Medicina de Botucatu da Universidade Estadual Paulista "Júlio de Mesquita Filho" UNESP - Botucatu (SP), Brasil.

4 Professora Substituta do Departamento de Neurologia, Psicologia e Psiquiatria da Faculdade de Medicina de Botucatu da Universidade Estadual Paulista "Júlio de Mesquita Filho" - UNESP - Botucatu (SP), Brasil.

${ }_{5}^{5}$ Professor Titular do Departamento de Ginecologia e Obstetrícia da Faculdade de Medicina de Botucatu da Universidade Estadual Paulista "Júlio de Mesquita Filho" - UNESP - Botucatu (SP), Brasil.

Apoio Financeiro: Programa Institucional de Bolsas de Iniciação Cientifica do Programa Institucional de Bolsas de Iniciação Científica do Conselho Nacional de Desenvolvimento Científico e Tecnológico (PIBIC/CNPq).

Bolsa de Iniciação Científica da Fundação de Amparo à Pesquisa do Estado de São Paulo - (FAPESP). 


\section{Introdução}

Apesar da expectativa social que associa o nascimento de uma criança a momentos de alegria e felicidade, o puerpério pode ser um período propenso a crises, visto que a gestação e o parto, muitas vezes, podem ser momentos estressantes na vida da mulher ${ }^{1}$. Esse momento pode ser particularmente estressante quando nasce uma criança com problemas determinados pela prematuridade, por doenças ou malformações.

Em pesquisa com mães de crianças prematuras foi demonstrado que, após o nascimento, $32 \%$ delas apresentavam sinais clínicos de ansiedade, e $14 \%$, de depressão ${ }^{2}$. A alteração do humor materno foi associado ao pobre desempenho motor e irritabilidade do recém-nascido ${ }^{3,4}$. Também foi verificado alto risco de estresse em mães de crianças com quadros de paralisia cerebral e doenças cardíacas congênitas ${ }^{5,6}$. Entretanto, esses resultados não são conclusivos. Estudos clínicos não constataram diferenças no estresse vivenciado por mães de crianças hospitalizadas com restrição de crescimento, quando comparadas com mães de crianças normais ${ }^{7}$. Uma metanálise das pesquisas realizadas no período de 1966 a 2005 com pais de crianças portadoras de espinha bífida constatou grande heterogeneidade no grau de ajustamento psicossocial dos pais ${ }^{8}$.

A inconsistência nos resultados pode estar relacionada ao fato de o estresse e a depressão materna serem fenômenos multideterminados. Ao lado das variáveis atribuídas ao quadro do recém-nascido, a combinação de diversos fatores adversos. como idade materna, baixo nível socioeconômico, baixo nível educacional, conflitos matrimoniais e falta de suporte paterno podem aumentar a vulnerabilidade da mãe e prejudicar seu estado emocional ${ }^{8-10}$. Estudo feito com mulheres após o primeiro mês de puerpério verificou que a ansiedade materna estava associada, significativamente, a intercorrências com o recém-nascido, especialmente no grupo de baixa renda ${ }^{11}$. Aumento nos níveis de estresse parental foram relacionados à sobrecarga e à insatisfação com os cuidados, ao isolamento social e às dificuldades financeiras, em uma amostra de pais de crianças com doenças cardíacas congênitas ${ }^{6}$.

Entretanto, deve-se assinalar que as variáveis ambientais nem sempre funcionam como fatores de risco para a instalação de quadros depressivos, podendo também funcionar como fatores de proteção. Uma pesquisa com mães cujos filhos eram portadores de mielomeningocele demonstrou que o grau da satisfação e de bem-estar dos pais estava altamente correlacionado à possibilidade de exercer algum controle sobre a situação, planejar e modificar o meio, usar procedimentos proativos e preventivos e poder contar com suporte social ${ }^{12}$. Há várias evidências na literatura demonstrando que o suporte social, bem como o suporte familiar, tem grande peso para a manutenção da saúde mental e enfrentamento de situações estressantes, além da adequação de comportamentos maternos em relação aos filhos ${ }^{13}$.

O estresse parental, especialmente a depressão materna, tem sido foco de preocupação dos pesquisadores pelos efeitos que pode provocar no desenvolvimento infantil, mesmo quando há remissão da patologia materna ${ }^{9,14,15}$. A depressão materna também está associada ao risco de abuso, bem como de trauma não intencional à criança ${ }^{16}$.

Apesar de a maioria dos estudos estarem centrados na depressão pós-parto, nos últimos anos algumas pesquisas se preocuparam com o estudo da ansiedade materna pela crença de que, na puérpera, os quadros ansiosos sejam mais frequentes que os quadros depressivos, embora sejam relativamente ignorados ${ }^{11,17,18}$. Sabe-se que a ansiedade e a depressão frequentemente coexistem, mas é preciso distingui-las de modo a oferecer tratamento apropriado $^{19}$.

Apesar do reduzido número de estudos sobre os efeitos da ansiedade materna no desenvolvimento do recém-nascido, foi constatado que a ansiedade no pósparto reduz a capacidade de enfrentamento, diminui a reatividade e sensibilidade e aumenta os sentimentos de ineficácia no desempenho do cuidado com a criança ${ }^{17}$. Existem evidências de que a ansiedade materna tem efeito negativo sobre a galactopoese e sobre a qualidade do vínculo mãe-bebê, o que justifica a adoção de medidas preventivas e terapêuticas ${ }^{11}$. As consequências da ansiedade materna não se limitam ao período do puerpério, mas têm efeitos em longo prazo. Mães com altos níveis de ansiedade no pós-parto, cinco anos após o nascimento da criança, apresentaram os indicadores mais numerosos de patologias psicossociais e de sinais de desadaptação de seus filhos quando comparadas a mães com baixos níveis de ansiedade no pós-parto ${ }^{20}$.

A grande maioria dos estudos sobre as consequências do nascimento de um filho com problemas sobre o estado emocional materno foram conduzidos com mães de crianças prematuras ${ }^{2,3}$. São poucos os trabalhos que contemplaram crianças nascidas com malformações ${ }^{5,6,8}$, especialmente no pós-parto imediato. A necessidade de melhor compreender os recursos e dificuldades emocionais maternas quando nasce um filho com malformações, para possibilitar ações preventivas e intervenções especificas, foi o objetivo deste estudo. Para tanto, foram comparados os sintomas de ansiedade e depressão de mães de recémnascidos com malformação aparente e de mães de recém nascidos eutróficos logo após o nascimento da criança.

\section{Métodos}

Foi realizado estudo prospectivo transversal para o qual foram convidadas a participar todas as puérperas cujos filhos 
eram portadores de malformações, internadas no período de Abril de 2005 a Março 2006, no Hospital das Clínicas da Faculdade de Medicina de Botucatu - Universidade Estadual Paulista "Júlio de Mesquita Filho" (FMB-UNESP). O projeto recebeu aprovação do Comitê de Ética em Pesquisa da FMB-UNESP. Todas as participantes assinaram o Termo de Consentimento Livre e Esclarecido.

O grupo de estudo foi constituído por 22 puérperas de recém-nascidos com malformações físicas aparentes que estavam internados na UTI ou UCI Neonatal (Grupo M), e o grupo controle, por 22 mães de recém-nascidos eutróficos (Grupo E) internados no berçário ou Alojamento Conjunto do Hospital das Clínicas da FMB-UNESP ou da Maternidade do Hospital Regional Sorocabana de Botucatu (Grupo E). Nos dois grupos, as mães foram estratificadas em três subgrupos segundo faixa etária (até 22; 23 a 32; e 33 a 42 anos) e paridade (primigesta, dois, três ou mais filhos). Nenhuma mãe era portadora de deficiência sensorial (visual ou auditiva) incapacitante, HIV, nem tinha distúrbios psiquiátricos ou síndromes genéticas descritas em prontuário.

Para avaliar o estado emocional foram utilizados o Inventário de Depressão de Beck (BDI) e o Inventário de Ansiedade Traço-Estado (IDATE). O BDI foi traduzido e adaptado para o Brasil por Cunha ${ }^{21}$. É uma escala de autoavaliação de manifestações comportamentais de depressão, porém, sem finalidade diagnóstica. Esse instrumento é composto por 21 categorias de sintomas e atitudes, com quatro ou cinco afirmações, cada uma refletindo um grau crescente de severidade de depressão (de 0 a 3 ).

O IDATE é um instrumento que pode ser utilizado para adultos normais como medida objetiva de estado (A-estado) e traço de ansiedade (A-traço), composto por 20 itens que o indivíduo deve responder avaliando a si mesmo em uma escala de quatro pontos. Os pontos são totalizados em um escore bruto que, por não permitir a comparação entre os indivíduos, é convertido para uma escala normalizada, de acordo com as normas do teste. $\mathrm{O}$ escore normalizado diferencia os indivíduos em uma hierarquia, ou seja, possibilita comparar o nível de ansiedade do indivíduo com a população média (normal) ${ }^{22}$.

Para a obtenção de informações sociodemográficas e informações clínicas foram consultados os prontuários médicos das mães e das crianças para determinar: condição de nascimento; evolução durante o período de internação; e condições de saúde da criança. No prontuário materno foram verificadas as condições de saúde pregressa, histórico gestacional e condições do parto. A avaliação materna ficou incompleta quando se tratava de mães transferidas de outros serviços.

Primeiramente, para a composição dos grupos, foram convidadas as mães que, após confirmação do diagnóstico pelo pediatra, diziam perceber as malformações de seus filhos e, paralelamente, mães com perfil similar cujos recém-nascidos haviam nascido eutróficos no mesmo período. Era realizada uma entrevista para coletar alguns dados sociodemográficos e, então, aplicados os testes. A fim de neutralizar a interferência da aplicação de um teste sobre o outro foi realizado um balanceamento, alterando a ordem de aplicação de cada instrumento de avaliação. A coleta de dados do grupo de estudo (Grupo M) ocorreu no hospital até sete dias após o nascimento, enquanto o recém-nascido encontrava-se internado na UTI ou UCI Neonatal. As mães do grupo controle (Grupo E) foram avaliadas no dia da alta hospitalar, entre dois e quatro dias após o nascimento.

Concomitantemente à realização do processo de avaliação das mães, os dados foram complementados com consultas aos prontuários médicos da criança e da mãe.

Os instrumentos de avaliação dos níveis de ansiedade e depressão foram corrigidos conforme as respectivas normas dos testes. Em seguida, foram analisadas as medidas de tendência central (medianas) dos escores obtidos pelas mães nas duas subescalas do IDATE (ansiedade-estado e ansiedade-traço) e no BDI. Para o cálculo das medianas dos escores maternos foram utilizados os escores normalizados no IDATE e os escores brutos no BDI, conforme as normas dos testes. Por fim, foi realizada a análise comparativa entre as medianas das pontuações do grupo de estudo e do grupo controle em cada uma das subescalas do IDATE (ansiedade-estado e ansiedade-traço) e no BDI, utilizandose o teste não-paramétrico U de Mann-Whitney para amostras independentes (comparação entre grupos).

Foi verificada, também, a distribuição das mães de acordo com escores indicativos de sintomas clínicos de ansiedade, disforia e depressão, quantificando-se o número de mães que apresentaram escores indicativos de sintomas clínicos. No IDATE foi utilizado como indicativo de sintomas de ansiedade em nível clínico o critério de corte de escore igual ou acima do percentil 75 , já utilizado em outro estudo ${ }^{2}$. Para o BDI, por sua vez, foram utilizados os critérios sugeridos para pacientes não diagnosticados com transtornos psiquiátricos, ou seja, pontuação acima de 15 , indicativa de disforia, e pontuação acima de 20 , indicativa de depressão $0^{23}$. Os resultados do grupo de estudo e do grupo controle foram comparados, em cada uma das subescalas, por meio do teste exato de Fisher e do teste do $\chi^{2}$.

A fim de verificar possíveis influências de variáveis intervenientes nos resultados, foram realizadas correlações entre as pontuações obtidas no IDATE e no BDI e as variáveis maternas (idade, número de filhos, escolaridade e estado civil) e, no Grupo M, com a localização corporal da malformação da criança a partir do teste de correlação de Spearman. Para o tratamento estatístico dos dados foi utilizado o SPSS 10.1 e estabeleceu-se o nível de significância de 0,05. 
Tabela 1 - Características das mães e de seus recém-nascidos

\begin{tabular}{|c|c|c|c|c|}
\hline Variáveis & \multicolumn{2}{|c|}{ Grupo M } & \multicolumn{2}{|c|}{ Grupo E } \\
\hline \multicolumn{5}{|l|}{ Variáveis maternas } \\
\hline Idade em anos - mediana (amplitude) & 24,5 & $16-41$ & 23,5 & $15-42$ \\
\hline \multicolumn{5}{|l|}{ Escolaridade - frequência (\%) } \\
\hline Ensino fundamental incompleto & 9 & 40,9 & 8 & 36,4 \\
\hline Ensino fundamental completo & 8 & 36,4 & 4 & 18,2 \\
\hline Ensino médio incompleto & 0 & - & 3 & 13,6 \\
\hline Ensino médio completo & 4 & 18,2 & 5 & 22,7 \\
\hline Ensino superior & 1 & 4,5 & 2 & 9,1 \\
\hline \multicolumn{5}{|l|}{ Filhos vivos - frequência (\%) } \\
\hline 1 filho & 10 & 45,5 & 10 & 45,5 \\
\hline 2 filhos & 5 & 22,7 & 5 & 22,7 \\
\hline 3 ou mais filhos & 7 & 31,8 & 7 & 31,8 \\
\hline \multicolumn{5}{|l|}{ Ocupação - frequência (\%) } \\
\hline Do lar & 14 & 63,6 & 15 & 68,2 \\
\hline Ocupações não qualificadas & 6 & 27,3 & 6 & 27,3 \\
\hline Ocupações não qualificadas & 2 & 9,1 & 1 & 4,5 \\
\hline \multicolumn{5}{|l|}{ Estado civil - frequência (\%) } \\
\hline União estável & 21 & 95,5 & 21 & 95,5 \\
\hline Solteira & 1 & 4,5 & 1 & 4,5 \\
\hline \multicolumn{5}{|l|}{ Variáveis do recém-nascido } \\
\hline $\begin{array}{l}\text { Idade gestacional em semanas - média } \\
\text { (desvio padrão) }\end{array}$ & 37 & 1,98 & 39 & 1,23 \\
\hline $\begin{array}{l}\text { Tempo de internação em dias - mediana } \\
\text { (amplitude) }\end{array}$ & 31 & $4-90$ & 2 & $2-4$ \\
\hline \multicolumn{5}{|l|}{ Tipo de malformação - frequência (\%) } \\
\hline SNC & 8 & 36,4 & & - \\
\hline Sistema Digestório & 5 & 22,7 & & - \\
\hline Outros & 9 & 40,9 & & - \\
\hline
\end{tabular}

Tabela 2 - Mediana, primeiro e terceiro quartis (entre colchetes) dos indicadores de ansiedade e depressão nas mães de recém-nascidos malformados (Grupo M) e eutróficos (Grupo E)

\begin{tabular}{lccc}
\hline \multirow{2}{*}{ Variável } & Grupo M & Grupo E & \multirow{2}{*}{ Valor de p } \\
\cline { 2 - 3 } & $(\mathrm{n}=22)$ & $(\mathrm{n}=22)$ & \\
\hline Disforia/Depressão (BDI) & $11,5[6,0 ; 20,0]$ & $7[5 ; 9]$ & 0,01 \\
Ansiedade-estado (IDATE) & $53,5[50 ; 61]$ & $46[40 ; 52]$ & 0,004 \\
Ansiedade-łraço (IDATE) & $52[47 ; 59]$ & $41[34 ; 47]$ & 0,001 \\
\hline
\end{tabular}

Teste U de Mann-Whitney.

Tabela 3 - Sintomas clínicos maternos de ansiedade-estado, ansiedade-traço e disforia/ depressão avaliados por meio do IDATE e do BDI ao nascimento da criança nos grupos $M$ $(n=22)$ e $E(n=22)$ : frequência ( $f)$ e porcentagem $(\%)$

\begin{tabular}{|c|c|c|c|c|c|}
\hline \multirow{3}{*}{ Sintomas clínicos } & \multirow{2}{*}{\multicolumn{2}{|c|}{$\begin{array}{c}\text { Grupo M } \\
(n=22)\end{array}$}} & \multirow{2}{*}{\multicolumn{2}{|c|}{$\begin{array}{c}\text { Grupo E } \\
(n=22)\end{array}$}} & \multirow{3}{*}{ Valor de $\mathrm{p}$} \\
\hline & & & & & \\
\hline & $f$ & $(\%)$ & $f$ & $(\%)$ & \\
\hline Disforia/Depressão (BDI) & 6 & $-27,2$ & 1 & $-4,5$ & $0,09 *$ \\
\hline Ansiedade-estado (IDATE) & 10 & $-45,4$ & 4 & $-18,1$ & $0,05^{\star \star}$ \\
\hline Ansiedade-traço (IDATE) & 8 & $-36,3$ & 0 & - & $0,003^{\star}$ \\
\hline
\end{tabular}

*Teste exato de Fisher; ${ }^{* *}$ teste do $\chi^{2}$.

\section{Resultados}

Ambos os grupos eram compostos por mães jovens, sendo que a idade mediana no grupo de estudo foi de 24 anos e, no Grupo Controle, de 23. Os grupos também se mostraram semelhantes quanto a situação conjugal, número de filhos e ocupação materna: $45 \%$ das mães eram primigestas e a maioria tinha uma união estável, sendo que apenas uma mãe de cada grupo não vivia com o pai do recém-nascido. Mais de $60 \%$ não exercia qualquer tipo de trabalho remunerado. Quanto à escolaridade, em ambos os grupos mais da metade das mães (77\% do Grupo M e 54\% do Grupo E) cursaram apenas o ensino fundamental, mas foi observada uma porcentagem maior de mães do grupo controle (32\%) que cursou o ensino médio ou superior em relação ao grupo de estudo (23\%) (Tabela 1).

Com relação aos recém-nascidos, notou-se que a idade gestacional média no grupo de estudo foi de 37 semanas e, no grupo controle, 39 semanas. Todas as crianças do Grupo M foram encaminhadas para a UTI logo após o nascimento, permanecendo hospitalizadas de quatro a 90 dias, enquanto a maioria das crianças do Grupo E se beneficiou do alojamento conjunto e o tempo de internação máxima foi de quatro dias. Em relação ao Grupo M, a maioria das malformações acometia o sistema nervoso central, como mielomeningocele e hidrocefalia (36\%), seguidas de malformações do aparelho digestivo: gastrosquise e onfalocele (23\%). Em 18\% dos casos havia malformações múltiplas, acompanhando síndromes como Down ou Edwards (Tabela 1). Houve ainda casos de hemangioma, higroma, teratoma, acondroplasia e fenda labial e palatina.

$\mathrm{Na}$ Tabela 2 se encontra a comparação das medianas dos escores de ansiedade (traço e estado) e disforia/depressão. O grupo de mães de recém-nascidos com malformação aparente (Grupo $\mathrm{M}$ ) apresentou índices significativamente maiores de depressão, ansiedade-traço e ansiedade-estado quando comparadas ao grupo de mães de recém-nascidos eutróficos (Grupo E).

Houve diferença significativa entre os escores indicativos de sintomas clínicos dos dois grupos: $27,3 \%$ das mães do Grupo $\mathrm{M}$ apresentaram escores indicativos para sintomas clínicos de disforia/depressão, em oposição a 4,5\% das mães do Grupo E. Apresentaram sintomas clínicos de ansiedade-estado 45,5\% das mães do Grupo M e 18,2 $\%$ das mães do Grupo E. Com relação à ansiedade-traço, 36,4\% das mães do Grupo M apresentaram sintomas clínicos, mas o resultado não aconteceu para nenhuma mãe do Grupo E (Tabela 3). Pode-se observar, também, que $22,7 \%$ de mães no Grupo M e 4,5\% de mães do Grupo E obtiveram escores indicativos, tanto para sintomas de depressão, como de ansiedade.

Não houve correlação significativa entre as pontuações nas duas escalas (IDATE e BDI) e variáveis maternas, tais como: idade, número de filhos, escolaridade, estado civil e a variável do recém-nascido (local da malformação). 


\section{Discussão}

A análise dos resultados mostrou que, logo após o nascimento, o grupo de mães cujos recém-nascidos apresentavam malformação aparente (Grupo $M$ ) apresentava índices significativamente maiores de depressão do que o grupo de mães de crianças eutróficas (Grupo E). Verificou-se, também, porcentagem significativamente maior de mães de crianças com malformações com sintomas clínicos de disforia/depressão do que mães de crianças eutróficas.

Estudos epidemiológicos estimam uma variação entre 10 a $20 \%$ nas taxas de depressão no pós-parto ${ }^{24}$. Pesquisa desenvolvida no Paquistão com mulheres jovens, provenientes de meio socioeconômico adverso encontrou taxas mais altas, isto é, um terço das puérperas sofria de depressão, sendo a maioria com sinais de depressão moderada e severa ${ }^{10}$. Essas diferenças tão marcantes podem ser explicadas pela diversidade de métodos de avaliação e critérios diagnósticos utilizados, bem como pelas diferenças econômicas e culturais dos diferentes grupos estudados ${ }^{24}$. Na presente pesquisa, a porcentagem de mães de recém-nascidos com malformações que apresentou sinais clínicos de depressão foi superior a de outros estudos brasileiros com puérperas ${ }^{24}$, mas inferior aos de pesquisa desenvolvida com mulheres atendidas pelo Programa Saúde da Família, em São Paulo, no qual foi encontrada prevalência de 37,1\% de depressão pós-parto. Os autores atribuem esses altos índices ao baixo nível socioeconômico da população estudada ${ }^{25}$.

Nesta pesquisa, a maioria das mães dos dois grupos tinha ensino fundamental incompleto e, as que trabalhavam, exerciam funções que requeriam pouca qualificação, características que, em alguns estudos epidemiológicos, podem ser consideradas indicativos de baixa renda ${ }^{25}$. No entanto, a porcentagem de mães de crianças eutróficas com sinais de depressão foi baixa $(4,5 \%)$, dando força à hipótese de que a alta prevalência de mães do Grupo M com sinais de depressão $(27,3 \%)$ esteja associada ao nascimento da criança com malformações.

Não foram encontrados estudos na literatura sobre depressão materna e malformação do recém-nascido, mas há pesquisas que constataram associação entre depressão puerperal e nascimento de crianças com outras doenças, como malformação cardíaca grave, spina bífida e atrasos no desenvolvimento ${ }^{5-8}$.

Os serviços de saúde têm grande preocupação com a depressão materna, especialmente pelas consequências para o desenvolvimento infantil. As mães deprimidas apresentam, de forma mais acentuada, impressões e sentimentos negativos com relação às crianças e à maternidade do que as mães sem depressão ${ }^{14}$. No geral, o estresse vivenciado por essas mães exacerba seus níveis de ansiedade e depressão e dificulta seu ajustamento e o desempenho de seu papel de cuidadora, na medida em que a depressão tende a afetar a disponibilidade cognitiva e emocional da mãe e, consequentemente, sua responsividade à criança ${ }^{9}$.

Os efeitos da depressão materna não se limitam ao atraso no desenvolvimento dos primeiros tempos de vida, mas podem ocasionar alterações na interação mãe-filho na primeira infância e causar prejuízos no desenvolvimento cognitivo e comportamental, em longo prazo. Um estudo realizado na Índia observou que a depressão materna esteve associada a um atraso no desenvolvimento cognitivo seis meses após o nascimento ${ }^{26}$.

Com relação à ansiedade, confirmando dados da literatura ${ }^{11,17}$, no presente estudo a porcentagem de mães com quadros ansiosos foi superior a de mães com sintomas de depressão. Ambos os grupos apresentaram sinais de ansiedade, mas as medianas dos escores de ansiedade-estado foram significativamente superiores no grupo das mães de crianças com malformações visíveis em comparação ao grupo de mães de crianças eutróficas. A presença de ansiedade no grupo de mães de recém-nascidos eutróficos era esperada, visto que ansiedade moderada, logo após o parto, é um fenômeno comum, na medida em que dificilmente a criança imaginária corresponde exatamente à criança real, exigindo dos pais uma adaptação que ocorre rapidamente, quando a mãe vai descobrindo suas competências e a própria criança exerce seu poder de sedução ${ }^{27}$. Em amostra não selecionada de mães de crianças saudáveis nascidas a termo, foi observado que $24,9 \%$ apresentaram ansiedade moderada após o parto, possivelmente associada à novidade do papel e às incertezas em desempenhá-lo a contento ${ }^{17}$.

A adaptação de mães de crianças que nascem doentes ou com problemas é mais difícil, especialmente frente a quadros graves que acarretam grande frustração, quebram expectativas relacionadas ao desempenho de maternidade $\mathrm{e}$ trazem mudanças na estrutura familiar. Essas dificuldades de adaptação podem explicar, nos dois grupos, a diferença significativa na porcentagem de mães que pontuaram para sinais clínicos de ansiedade.

Os índices de ansiedade pós-parto das mães de recémnascidos com malformações podem ter sido influenciados, inicialmente, pelas características da criança e pelas perspectivas sobre sua condição de vida futura. Em um estudo de corte transversal com mulheres atendidas em clínica privada, após análise multivariada, observou-se que a ansiedade-traço e estado se associavam a intercorrências com o recém-nascido ${ }^{11}$. Mães de crianças que tinham spina bífida apresentaram níveis de estresse superiores aos dos pais. Mostravam preocupação com as futuras dificuldades de locomoção e comunicação da criança. A saúde mental de ambos tinha forte associação com a existência de suporte familiar, assim como a adaptação dependia da qualidade das relações do casal ${ }^{8}$. No caso de crianças com malformações 
visíveis, especialmente com defeitos que atingem a face, a essas preocupações se somam o medo dos preconceitos e a preocupação com sua inserção social ${ }^{28}$.

Os níveis de ansiedade identificados nas mães de recém-nascidos com malformações podem também estar associados a traços de personalidade, visto que a ansiedade-traço era significativamente maior nas mães de crianças malformadas que nas mães de eutróficos. A ansiedade-traço diz respeito a diferenças individuais, traços relativamente estáveis de personalidade, uma tendência a reagir de forma ansiosa diante de situações percebidas pelo indivíduo como ameaçadoras ${ }^{22}$. O puerpério parece ser um período de maior risco para o surgimento ou piora de distúrbios ansiosos que, nessa fase, apresentam características particulares e causam problemas específicos ${ }^{11}$. Tal constatação insere essas mães em grupo de risco, na medida em que apresentam mais propensão a reagir de maneira ansiosa frente a situações estressantes, como o nascimento de uma criança com problemas.

O encaminhamento de todas as crianças com malformações para a UTI pode ter agravado a ansiedade e a depressão materna. Há evidências que mostram que os níveis de ansiedade de mães que percebiam que ela própria ou a criança precisava permanecer mais tempo no hospital eram superiores àqueles de mães que recebiam alta hospitalar juntamente com o filho ${ }^{17}$. Condições patológicas do recém-nascido e prolongamento da internação da criança foram identificadas como fatores de risco para depressão pós-parto ${ }^{18}$.

A hospitalização do filho, independentemente do motivo, constitui uma situação estressante para as mães. Porém, algumas particularidades podem estar associadas às diferentes patologias. Estudos realizados com mães de prematuros mostraram níveis mais elevados de ansiedade durante a internação hospitalar em comparação ao período pós-alta ${ }^{2,29}$. As mães de prematuros também apresentam mais sintomas de ansiedade quando comparadas com mães de recém-nascidos a termo. Porém, não houve diferença significativa quanto aos sintomas depressivos nos dois grupos ${ }^{2}$. No presente estudo, por outro lado, com bebês de malformações visíveis houve diferenças significativas, tanto nos sintomas ansiosos, quanto nos depressivos.

A hospitalização da criança coloca a mãe frente ao dilema de ter de escolher entre permanecer com o filho, atender às demandas familiares e às suas próprias necessidades, acrescidas pela exigência de se adaptar a uma nova dinâmica determinada por rotinas e normas institucionais ${ }^{30}$. Além das angústias geradas pelas condições de saúde das crianças, já que no ambiente hospitalar os cuidados ficam e a criança tem pouco contato direto com a mãe, aumenta sua percepção de inadequação para os cuidados.
A comorbidade é outro aspecto que merece destaque. Vinte e dois por cento de mães de recém-nascidos com malformações e $4 \%$ de mães de recém-nascidos eutróficos apresentaram tanto sinais clínicos de ansiedade como de depressão. A superposição entre sintomas de ansiedade e depressão e a consequente dificuldade para a avaliação desses sintomas separadamente pode ter várias explicações. Em primeiro lugar, pode ser efeito de limitações psicométricas das escalas utilizadas para a avaliação, em especial do IDATE e do BDI, utilizados no presente estudo, que apresentam alta correlação. Outra hipótese é que os dois construtos, ansiedade e depressão, podem ser componentes de um processo de estresse psicológico geral. Por fim, a ansiedade e a depressão, além de características específicas de cada construto, podem apresentar um componente de sobreposição, composto por sintomas inespecíficos de ambos os construtos: o afeto negativo, que congrega vários estados aversivos, como constrangimento, raiva, culpa medo, tristeza, preocupação, entre outros ${ }^{31}$.

Apesar de os dados desta pesquisa terem sido obtidos a partir de instrumentos de autoavaliação que apresentam algumas limitações quanto à confiabilidade das informações fornecidas pelo sujeito ${ }^{31}$, a porcentagem de mães com escores indicativos para ansiedade, depressão e ambos sugere a necessidade de suporte pós-natal, seja individual ou grupal, para amenizar os efeitos adversos dos estressores no desenvolvimento da criança e atender a necessidade das mães. O suporte psicológico em grupo oferecido às mães por alguns serviços hospitalares durante o período de hospitalização do recém-nascido diminui os níveis de ansiedade e depressão materna, ao menos para as mães de crianças pré-termo de muito baixo peso ${ }^{32}$.

Uma limitação do estudo aconteceu devido ao tamanho amostral. Apesar de se escolher um hospital de referência, que atende uma grande área populacional, houve dificuldades para compor a amostra: a incidência dos casos é esporádica e há um grande número de óbitos. Houve a tentativa de se contornar o problema desenvolvendo o trabalho em um hospital-escola de nível terciário, que conta com uma UTI de berçário para onde são encaminhados os recém-nascidos de alto risco da região. É um serviço de medicina fetal de referência que recebe as grávidas portadoras de fetos com malformação que, em geral, acabam dando a luz neste hospital. Por outro lado, a tentativa de garantir que as crianças do grupo das eutróficas tivessem nascido na mesma época das crianças do grupo com malformações não permitiu um sorteio aleatório da amostra.

O número reduzido de sujeitos com quadros bastante heterogêneos, tanto em relação à visibilidade quanto à gravidade, pode ter prejudicado, por exemplo, a identificação de variáveis associadas aos indicadores emocionais, como: 
idade, número de filhos, tipo de malformação etc. Para que se obtenham dados que permitam análises estatísticas mais consistentes, sugere-se que, nos próximos estudos, seja programada a composição de amostras multicêntricas, assim como ocorre em outros estudos de síndromes de rara aparição.

\section{Referências}

1. Zelkowitz P, Papageorgiou A. Maternal anxiety: an emerging prognostic factor in neonatology. Acta Paediatr. 2005;94(12): 1704-5.

2. Padovani $F H$, Carvalho $A E$, Duarte $G$, Martinez FE, Linhares $M B$. Anxiety, dysphoria, and depression symptoms in mothers of preterm infants. Psychol Rep. 2009; 104(2):667-79.

3. Murray L, Stanley C, Hooper R, King F, Fiori-Cowley A. The role of infant factors in postnatal depression and mother-infant interactions. Dev Med Child Neurol. 1996;38(2):109-19.

4. Black MM, Baqui AH, Zaman K, El Arifeen S, Black RE. Maternal depressive symptoms and infant growth in rural Bangladesh. Am J Clin Nutr. 2009;89(3):951S-7S.

5. Manuel J, Naughton M, Balkrishnan R, Paterson Smith B, Koman LA. Stress and adaptation in mothers of children with cerebral palsy. J Pediatr Psychol. 2003;28(3):197-201.

6. Lawoko S, Soares JJ. Psychosocial morbidity among parents of children with congenital heart disease: a prospective longitudinal study. Heart Lung. 2006;35(5):301-14.

7. Singer LT, Salvator A, Guo S, Collin M, Lilien L, Bayley J. Maternal psychological distress and parenting stress after the birth of a very low-birth-weight infant. JAMA. 1999;281(9): 799-805.

8. Vermaes IP, Janssens JM, Bosman AM, Gerris JR. Parents' psychological adjustment in families of children with spina bifida: a meta-analysis. BMC Pediatr. 2005;5:32.

9. Frizzo GB, Piccinini CA. Interação mãe-bebê em contexto de depressão materna: aspectos teóricos e empíricos. Psicol Estud. 2005; 10(1):47-55.

10. Muneer A, Minhas FA, Tamiz-ud-Din Nizami A, Mujeeb F, Usmani AT. Frequency and associated factors for postnatal depression. J Coll Physicians Surg Pak. 2009; 19(4):236-9.

11. Faisal-Cury A, Menezes PR. Ansiedade no puerpério: prevalência e fatores de risco. Rev Bras Ginecol Obstet. 2006;28(3): $171-8$.

12. Fagan J, Schor D. Mothers of children with spina bifida: factors related to maternal psychosocial functioning. Am J Orthopsychiatry. 1993;63(1):146-52

13. Cruz EBS, Simões GL, Faisal-Cury A. Rastreamento da depressão pós-parto em mulheres atendidas pelo Programa de Saúde da Família. Rev Bras Ginecol Obstet. 2005;27(4):181-8.

14. Schwengber DDS, Piccinini CA. A experiência da maternidade no contexto da depressão materna no final do primeiro ano de vida do bebê. Estud Psicol. 2005;22(2):143-56.

15. Engle PL. Maternal mental health: program and policy implications. Am J Clin Nutr. 2009;89(3):963S-6S.

16. Neeldman R, Walders N, Kelly S, Higgins J, Sofranko K, Drotar D. Impacto da triagem para depressão materna em uma clínica pediátrica: um estudo exploratório (M.M.G.B. Mataloun, Trad.). Correios SBP. 2000;6:18-9.
17. Britton JR. Pre-discharge anxiety among mothers of well newborns: prevalence and correlates. Acta Paediatr. 2005;94(12):1771-6.

18. Evans GM, Vicuña MM, Marin R. Depresion postparto realidad en el sistema publico de atención de salud. Rev Chil Obstet Ginecol. 2003;68(6):491-4.

19. Miller RL, Pallant JF, Negri LM. Anxiety and stress in the postpartum: is there more to postnatal distress than depression? BMC Psychiatry. 2006:6:12

20. Barnett B, Schaafsma MF, Guzman AM, Parker GB. Maternal anxiety: a 5-year review of an intervention study. J Child Psychol Psychiatry. 1991;32(3):423-38.

21. Cunha JA. Manual da versão em português das Escalas Beck. São Paulo: Casa do Psicólogo; 2001.

22. Spielberger CD, Biaggio A, Natalício LF. Inventário de ansiedade traço estado: manual de psicologia aplicada. Rio de Janeiro: Cepa; 1979

23. Ingram RE, Kendall PC, Smith TW, Donnell C, Ronan K. Cognitive specificity in emotional distress. J Pers Soc Psychol. 1987;53(4): 734-42.

24. Moraes IGS, Pinheiro RT, Silva RA, Horta BL, Sousa PLR, Faria $A D$. Prevalência da depressão pós-parto e fatores associados. Rev Saúde Pública. 2006;40(1):65-70.

25. França E, Souza JM, Guimarães MDC, Goulart EMA, Colosimo $E$, Antunes CMF. Associação entre fatores sócio-econômicos e mortalidade infantil por diarréia, pneumonia e desnutrição em região metropolitana do sudeste do Brasil: um estudo caso-controle. Cad Saúde Pública. 2001;17(6):1437-47.

26. Patel V, DeSouza N, Rodrigues M. Postnatal depression and infant growth and development in low income countries: a cohort study from Goa, India. Arch Dis Child. 2003;88(1):34-7.

27. Klaus MH, Kennel JH. Pais-bebê: a formação do apego. Porto Alegre: Artes Médicas; 1993.

28. Fernandes AY, Ducati LG, Silva MV, Abramides DVM, Perosa GB, Palhares $A$, et al. Síndrome de crouzon: fatores envolvidos no desenvolvimento neuropsicológico e na qualidade de vida. Arq Neuropsiquiatr. 2007;65(2b):467-71

29. Zanardo V, Freato F. Home oxygen therapy in infants with bronchopulmonary dysplasia: assessment of parental anxiety. Early Hum Dev. 2001;65(1):39-46.

30. Dittz ES, Mota JAC, Sena RR. O cotidiano no alojamento materno, das mães de crianças internadas em uma Unidade de Terapia Intensiva Neonatal. Rev Bras Saúde Matern Infant. 2008;8(1):75-81.

31 . Gorenstein C, Andrade L, Zuardi AW. Escalas de Avaliação Clínica em Psiquiatria e Psicofarmacologia. São Paulo: Lemos Editorial; 2000.

32. Carvalho AE, Linhares MB, Padovani FH, Martinez FE. Anxiety and depression in mothers of preterm infants and psychological intervention during hospitalization in neonatal ICU. Span J Psychol. 2009;12(1):161-70 